\title{
Erratum: Is the Voter Model a Model for Voters? [Phys. Rev. Lett. 112, 158701 (2014)]
}

\author{
Juan Fernández-Gracia, Krzysztof Suchecki, José J. Ramasco, Maxi San Miguel, and Víctor M. Eguíluz
}

(Received 28 July 2014; published 18 August 2014)

DOI: 10.1103/PhysRevLett.113.089903

PACS numbers: 89.65.Ef, 05.40.Ca, 89.75.Fb, 89.75.Hc, 99.10.Cd

The horizontal axis of Fig. 2(a) of the Letter was wrongly scaled. The correct Fig. 2(a) is presented below. This affects the assessment of the critical value $D^{*}$ but not any other statement or conclusion drawn in the Letter.

Summary of corrections:

Figure 2(a) of the original manuscript should look as Fig. 2 of this communication.

Page 3, left column should say: "[...]Best agreement is obtained for $D=0.02[\ldots]$ For $D=0.02$ the standard deviation of the vote-share distribution[...]"

Page 3, right column should say: "[...]Sets of electoral results are produced with the model, with $D=0.02$ and with a fixed number of Monte Carlo steps between elections.[...]"

Caption of Fig. 3 should start: "Parameters of the simulation are $\alpha=1 / 2, D=0.02[\ldots]$ "

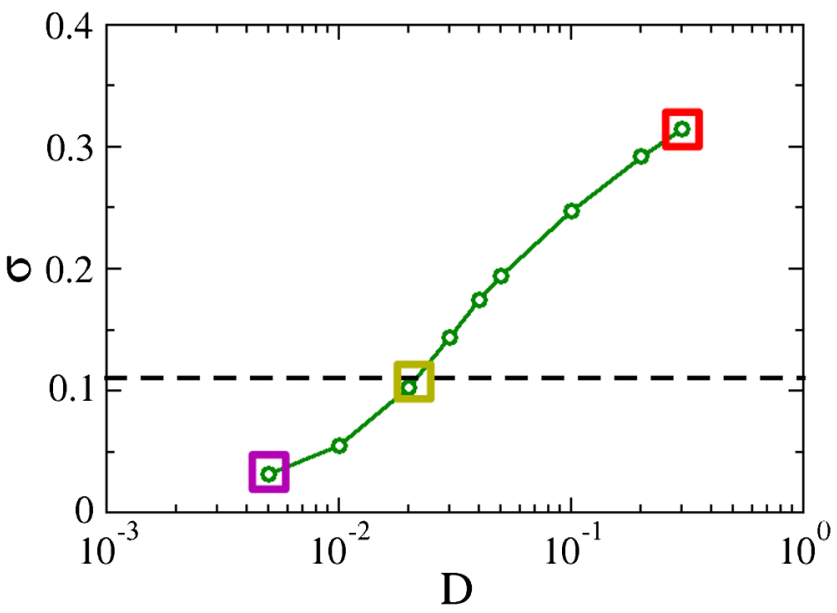

FIG. 2 (color online). (a) Vote-share standard deviation versus noise intensity $D$. The dashed black line marks the dispersion of the empirical data $\left(\sigma_{e}=0.11\right)$. Boxes surrounding the main plot (not shown here, they stay the same as in the Letter) display results obtained with the level of noise marked as squares and include the distribution of vote-shares shifted to have zero mean, and their spatial correlations. Black curves are initial conditions. In the red box, the red curve is for $10 \mathrm{MC}$ steps, and the orange for $20 \mathrm{MC}$ steps; in the green box, the times are $100 \mathrm{MC}$ steps (red) and $200 \mathrm{MC}$ steps (orange); in the purple box, $40 \mathrm{MC}$ steps (red) and $140 \mathrm{MC}$ steps (orange). 